\section{$\underset{\substack{\text { hommes } \\ \text { \& migrations }}}{ }$}

\section{Hommes \& migrations}

Revue française de référence sur les dynamiques

migratoires

\section{$1322 \mid 2018$}

Exposer les migrations

\title{
Exposer le fait migratoire : l'expérience du Musée dauphinois
}

\section{Olivier Cogne}

\section{Q OpenEdition \\ Journals}

Édition électronique

URL : https://journals.openedition.org/hommesmigrations/6662

DOI : 10.4000/hommesmigrations.6662

ISSN : 2262-3353

\section{Éditeur}

Musée national de l'histoire de l'immigration

\section{Édition imprimée}

Date de publication : 1 juillet 2018

Pagination : 83-89

ISBN : 978-2-919040-42-1

ISSN : $1142-852 X$

Référence électronique

Olivier Cogne, «Exposer le fait migratoire : l'expérience du Musée dauphinois », Hommes \& migrations [En ligne], 1322 | 2018, mis en ligne le 01 juillet 2020, consulté le 21 janvier 2022. URL : http:// journals.openedition.org/hommesmigrations/6662 ; DOI : https://doi.org/10.4000/ hommesmigrations.6662 


\title{
EXPOSER LE FAIT MIGRATOIRE : L'EXPÉRIENCE DU MUSÉE DAUPHINOIS
}

Par OLIVIER COGNE, directeur du Musée dauphinois.

\author{
Lieu de connaissance de I'histoire alpine, le Musée dauphinois \\ est aussi un espace de réflexion sur le vivre ensemble \\ et s'inscrit dans une relation de proximité avec les habitants \\ de son territoire. Fidèle à la démarche participative qui est I'un \\ des piliers de son travail muséographique, le musée collecte \\ et rassemble des objets et des témoignages qui confèrent \\ à chacune de ses expositions la forme d'une mémoire collective \\ partagée. Depuis les années 1980, le Musée dauphinois \\ a fait place entre autres aux Grecs, aux Italiens, aux Arméniens, \\ aux Maghrébins ou aux rapatriés d'Algérie pour refléter \\ la diversité culturelle des populations du département.
}

Le Musée dauphinois s'est affirmé au fil du temps comme un établissement ouvert sans restriction aux cultures de tous horizons. À l'inverse d'une approche strictement locale qui pourrait le réduire à n'être qu'un musée d'histoire et d'ethnographie régionale, il revendique son attachement à défendre la diversité culturelle à une époque où la mondialisation des échanges tend plutôt à l'uniformisation des pratiques.

Pour autant, s'il n'est pas qu'un musée du Dauphiné ou de l'Isère ${ }^{1}$, il entend interroger le territoire qui l'abrite pour souligner son brassage culturel. Cette orientation a été impulsée par la municipalité d'Hubert Dubedout qui remporte les élections en mars 1965 et plus particulièrement par Bernard Gilman, adjoint aux affaires culturelles. Dans une équipe située à gauche de l'échiquier politique, lélu s'appuie notamment sur son expérience acquise au sein du mouvement d'éducation populaire Peuple et culture $^{2}$ pour engager une politique culturelle innovante qui confère à Grenoble la qualité de ville laboratoire. Saisissant l'opportunité des Jeux olympiques d'hiver de 1968, la municipalité obtient des moyens importants de l'État - à la faveur de la manifestation sportive internationale - pour ouvrir la Maison de la culture et rénover le Musée dauphinois. Bernard Gilman résume ainsi la philosophie qu'il souhaite donner à l'établissement muséal : un lieu qui permette de "situer dans leur contexte historique les problèmes actuels de la région ${ }^{3}$ ». Une approche qui n'a pas été remise en cause depuis, quels que soient les changements institutionnels ${ }^{4}$ et politiques. 


\section{Un long cycle d'expositions dédiées aux migrations}

C'est principalement par les expositions temporaires que la démarche d'ouverture du Musée dauphinois se manifeste et, plus précisément, à travers celles qu'il consacre depuis trente-cinq ans environ aux Isérois venus d'ailleurs, faisant figure de pionnier en France en la matière ${ }^{5}$.

Dès 1982, une première exposition témoigne de la volonté du musée de faire place à l'histoire des hommes et des femmes qui, arrivés de l'étranger, se sont installés dans ce département durant l'époque contemporaine. La démarche n'est pas courante alors et l'exposition Le Roman des Grenoblois ${ }^{6}$ - c'est son nom - provoque quelques réactions étonnées, voire très critiques ${ }^{7}$. Il n'est pas habituel de souligner dans le récit local la diversité des origines de la population. Les conservateurs du musée ${ }^{8}$ font, en effet, le choix d'évoquer la migration italienne, puis algérienne, comme des éléments constitutifs des identités grenobloises. En s'appuyant sur des récits de vie fictifs, mais basés sur des faits sociaux bien réels, ils mettent en scène deux familles qui répondent aux noms génériques de Sebastiano et Kaled. Racontant leurs parcours de migrants et leurs installations en Isère, ces personnages ne masquent rien des difficultés auxquelles ils ont été et sont confrontés.

Globalement, l'approche semble bien comprise par le public et personne ou presque ne trouve à redire lorsque le musée présente en 1988 Corato-Grenoble. Cette fois, tandis que les deux localités viennent de se jumeler, c'est toute une exposition qui est consacrée aux nombreux Isérois originaires de la petite ville italienne des Pouilles. Sans le savoir alors, le musée engage un cycle d'expositions sur le fait migratoire qu'il poursuit toujours. Près d'une dizaine de manifestations se succèdent autour des liens que ce département entretient grâce à sa population avec différents pays du monde, pour ne pas dire tous... au regard de la diversité des origines des habitants d'un territoire qui compte aujourd'hui près d'un million deux cent cinquante mille personnes.

\section{Des Grecs de Grenoble à D'Isère et d'Arménie}

L'origine de ces projets est multiple. Mais ils répondent la plupart du temps à une demande d'habitants de l'Isère soucieux de faire connaitre l'histoire dont ils sont issus. À l'exposition Des Grecs de Grenoble ${ }^{9}$, qui relate en 1993 le parcours de populations exilées d'Asie mineure et arrivées en Isère au début du siècle dernier, fait écho en 1997 D'Isère et d'Arménie ${ }^{10}$ qui émane d'associations arméniennes. Ce projet s'inscrit dans un contexte politique, celui de la reconnaissance officielle par la France du génocide perpétré par le gouvernement des Jeunes-Turcs en 1915 et 1916 . L'exposition suscite à l'époque une réaction critique des autorités consulaires turques. Mais elle est saluée par des parlementaires isérois dont certains voteront quelques années plus tard la loi tant attendue. Le moment est aussi marqué par la présence des derniers rescapés du génocide dont la mémoire est recueillie par l'équipe du musée. Cette collecte de récits de vie sera systématisée pour l'ensemble des projets ${ }^{11}$. Car la mémoire permet d'humaniser et d'incarner un propos en plus des éléments généraux proposés aux visiteurs. Elle a valeur d'archive au-delà de la présentation des expositions.

5. Jean-Claude Duclos, "L'immigration au Musée dauphinois ", in Écarts d'identité, n 108, 2006, pp. 16-26. 6. Un livre accompagne cette exposition : Le Roman des Grenoblois : 1840-1980, en 6 actes et 44 tableaux, Grenoble, Grenoble animation information, 1982. 7. Jean-Claude Duclos évoque ainsi des lettres anonymes adressées au musée appelant à ce que soit retiré le personnage fictif d'Ahmed Kaled, Algérien d'origine, en jugeant sa présence déplacée dans l'exposition : "De l'immigration au Musée dauphinois ", op. cit., pp. 97-98. 8. Il importe de citer comme acteurs de ce projet : Jean-Pierre Laurent, directeur du musée de 1971 à 1986, et Jean Guibal, conservateur-adjoint. Ce dernier prend la tête du musée de 1986 à 2000 et assume les mêmes fonctions de 2011 à 2016 . Son rôle et celui d'un autre conservateur, Jean-Claude Duclos (lui-même directeur du musée de 2000 à 2011), sont essentiels dans les expositions abordées ici. 9. Nous renvoyons au livre de l'exposition : Jean-Claude Duclos (dir.), Des Grecs: les Grecs de Grenoble, les costumes de la Grèce traditionnelle, Grenoble, Musée dauphinois, 1993. 10. Jean-Claude Duclos (dir.), D'Isère et d'Arménie : histoire d'une communauté, Grenoble, Musée dauphinois, 1997. 11. Recueillis sur un support audio ou audiovisuel, ces témoignages sont conservés par le service de documentation du musée et accessibles aux chercheurs à la condition d'un accord des témoins ou de leurs ayants droit. 


\section{D'Isère et du Maghreb à Français d'Isère et d'Algérie}

En 1999, c'est la mémoire des Maghrébins que le musée choisit de questionner avec l'histoire des populations nord-africaines arrivées en Isère à partir des années 1950 ; l'une des principales migrations que ce département ait connue. Naturellement, la colonisation est au cœur du sujet et, en particulier, la guerre d'Algérie dont l'évocation suscite encore aujourd'hui tant de débats et de crispations.
Tous ces projets sont bâtis avec le concours d'experts issus pour la plupart de l'université et choisis en raison de leurs travaux afin de garantir autant que possible l'objectivité et la scientificité du propos. La démarche diffère ici d'autres structures muséales qui ont l'habitude de confier le commissariat d'exposition à des chercheurs patentés. Tout en s'entourant d'experts, le Musée dauphinois tient à conserver la maîtrise de son ouvrage jusqu'à la rédaction des textes qu'il considère comme le médium central de son discours muséographique. La caution scientifique reste, cependant, nécessaire 
pour aborder ces sujets dont on connaît la sensibilité. Les réactions ne manquent pas, positives ou négatives. La plus violente d'entre elles fut celle d'un commando d'extrême droite qui fit irruption dans la chapelle du musée un soir de novembre 1999 pour empêcher l'intervention de l'historien algérien Mohammed Harbi ; la presse nationale s'en fera l'écho. Courageusement, après cet incident, le conférencier poursuivra son propos sur les rapports entre colonisation et immigration.

De l'exposition D'Isère et du Maghreb ${ }^{12}$ naît un autre projet dédié à la mémoire des Français d'Algérie. De la rencontre de Jean-Claude Duclos, directeur du musée, avec les associations iséroises d'anciens rapatriés - soucieuses à leur tour d'être entendues germe, en effet, l'idée de raconter l'histoire douloureuse de leur départ d'Afrique du Nord. Tenter de concilier des mémoires conflictuelles: tel est l'objectif ambitieux du musée à travers ces deux projets mobilisant des acteurs

Tout en s'entourant d'experts,

le Musée dauphinois tient à conserver la maîtrise de son ouvrage jusqu'à la rédaction des textes qu'il considère comme le médium central de son discours muséographique.

\section{dont la perception de la présence} française en Algérie à l'époque coloniale est souvent radicalement différente. La démarche est complexe mais repose sur le vécu d'individus désireux d'exprimer leur amour d'une terre qui fut aussi la leur, une partie de leur existence. Elle aboutit en 2003 à un travail qui semble faire consensus auprès des populations les plus concernées, tout en se gardant bien d'idéaliser le passé colonial : Français d'Isère et d'Algérie ${ }^{13}$. Au-delà des antagonismes forts qui perdurent cinquante-cinq ans après la fin du conflit, l'exposition parvient à un équilibre autour de textes d'Albert Camus exprimant son attachement à la terre qui l'a vu naître ${ }^{14}$. De la colonisation, il en sera encore question quelques années plus tard dans le cadre de l'exposition Ce que nous devons à l'Afrique ${ }^{15}$ dédiée aux rapports que l'Occident et notamment la France entretiennent avec le continent. Changer le regard, tel est souvent le moteur de ces projets. Celui-ci ne fait pas exception en rappelant dans son introduction que les premiers hommes sont apparus en Afrique et en soumettant à la sagacité des visiteurs cette réflexion : ne sommes-nous pas tous originaires de l'Afrique?

\section{Un air d'Italie}

Le musée se tourne à nouveau vers l'Italie en $2011^{16}$. Cette fois, il n'est plus seulement question des liens entre Corato et Grenoble, mais des rapports de toute la péninsule avec l'Isère qui est l'un des principaux départements où les migrants italiens se sont installés. L'exposition du musée constitue le temps fort d'une "Année de l'Italie » à laquelle concourent de nombreux acteurs culturels. L'exposition témoigne notamment de l'italianité ostensible dans certains quartiers de Grenoble et de celle, plus sensible, qui fait dire à des Isérois d'origine italienne leur attachement de cœur à la péninsule et à ses cultures. Des pratiques culturelles que les habitants de ce département ont depuis longtemps faites leur, quelles que soient leur origines, et dont ne manque pas de faire état l'exposition en invoquant la cuisine "della mamma » qui a envahi les assiettes ou les supporters de la « Juve $»^{17}$. Derrière «l'intégration réussie » de ces habitants se cache comme souvent une histoire plus complexe marquée par une italophobie que beaucoup semblent avoir oubliée, mais dont se souviennent les plus anciens lorsqu'ils ont dû faire leur place dans la société française; la mémoire des «Macaronis » n'est pas éteinte. L'exposition interroge aussi la condition des migrants à travers le temps et la tragédie du déracinement qui relève moins d'un choix que d'une nécessité pour survivre. 
La mise en parallèle dans la scénographie de deux images à un siècle d'intervalle, celle d'Italiens en attente du départ dans le port de Gênes et celle de Nord-Africains à leur arrivée à Lampedusa - l'une des «portes » de l'Europe -, gage du sort dramatique des populations migrantes et de l'incapacité des États à y remédier. L'exposition fournit aussi l'occasion au musée d'interroger son public à l'issue de la visite avec une question volontairement provocatrice: "Des expositions sur des populations d'origine étrangère ont-elles, selon vous, leur place dans un musée de patrimoine régional ? " Les réponses recueillies au moyen de post-it laissés à la libre disposition des visiteurs viennent dans leur très grande majorité conforter le musée dans sa démarche.

\section{Tsiganes : la vie de bohème?}

La situation des populations « roms » vivant en France, et notamment dans la région, et la stigmatisation grandissante dont elles sont l'objet incitent le musée à engager quelques années plus tard une nouvelle exposition qui répond au titre de : Tsiganes. La vie de bohème ${ }^{18}$ ? Les lieux de vie de ces migrants ne sont pas sans rappeler les bidonvilles dans lesquels vivaient les travailleurs algériens en France dans les années 1950 et 1960 et que l'on pensait révolus. Le moment paraît d'autant plus propice pour engager ce projet que le Conseil général de l'Isère, dont relève le Musée dauphinois, vient d'établir un rapport sur la situation des familles "roms" dans l'agglomération grenobloise. Marie-Colette Lalire, auteure de ce document et ancienne attachée auprès de l'ambassade de France en Roumanie, compte aussi parmi les membres de l'association locale Roms Action qui jouera un rôle essentiel dans cette opération.

Le propos de l'exposition évoque à la fois les migrants venus ces dernières années de l'Est de l'Europe, principalement de Roumanie, mais aussi la présence séculaire de populations de culture tsigane en Isère et françaises depuis des générations. Les amalgames sont nombreux et tendent à présenter une vision uniforme de groupes qui se caractérisent en réalité par une incroyable diversité à l'échelle européenne. L'un des enjeux de ce projet est de redonner du sens aux mots face à l'usage de termes employés par les " gadjé » ${ }^{19}$ qui contribuent à entretenir les confusions et l'incompréhension. L'exposition revient également sur les préjugés tenaces qui visent ces populations et participent depuis des siècles à leur mise au ban des sociétés, jusqu'au génocide perpétré par les nazis durant la Seconde Guerre mondiale. Loin d'être passée, la mise au ban dont ils sont l'objet semble même ravivée ces dernières années un peu partout en Europe.

A contrario, l'exposition souligne la fascination que ces populations suscitent à travers la peinture ou l'œil des photographes. Point de vision misérabiliste, mais au contraire un regard qui souligne la beauté de leur culture. Le portrait de La Zingara réalisé par le peintre Ernest Hébert en est l'un des exemples. L'exposition s'achève en abordant l'incroyable capacité de résistance des populations tsiganes dans la préservation de leurs modes de vie qui, échappant à la norme, sont mis à mal depuis des siècles.

\section{La démarche participative}

De tels projets d'exposition n'ont de sens que s'ils associent les populations les plus concernées afin de « ne pas parler en lieu et place de ». Est-il envisageable, en effet, d'aborder l'histoire et les cultures des populations tsiganes dans un musée sans ces dernières ? Pour autant, de telles pratiques ne sont pas si fréquentes dans les musées français quand elles sont devenues quasi incontournables chez bon nombre de nos collègues nord-américains, notamment. Elles supposent d'élargir les conseils scientifiques des expositions à des membres qui ne seraient pas invités en leur qualité d'experts, mais 
en tant qu'acteurs-témoins d'une expérience de vie et de pratiques culturelles. C'est en tout cas le parti pris du Musée dauphinois qui, depuis les années 1980, réunit des personnes d'horizons divers, qu'elles soient issues du monde « savant » ou de la société civile. La confrontation de ces regards, rarement associés, a grandement contribué à nourrir ces projets en offrant à chacun la possibilité d'entendre le point de vue de l'autre au sein d'une seule et même assemblée. Aucune hiérarchie ici dans les prises de parole. C'est au musée qu'il revient le difficile exercice de synthèse pour préciser les contours de l'exposition : les principaux thèmes, les idées-force, les valeurs portées.
La tâche n'est pas simple, car elle suppose parfois de pouvoir convaincre des personnes qui ne sont jamais entrées dans un musée et dont le quotidien est aux antipodes de la préparation d'une exposition. Le concours des associations, en particulier investies dans le champ social, fut déterminant dans le cas du projet Tsiganes pour convaincre quelques familles «roms » d'y participer. Des acteurs associatifs locaux que le musée ne manque pas d'inviter dans le cadre de ce qui ne convient plus vraiment d'appeler un conseil scientifique, mais plus sobrement un groupe de travail. Le rôle des associations à caractère mémoriel, culturel et social, ou encore de ressortissants des pays concernés par 
les expositions est essentiel dans la lecture globale des projets et dans leur mise en œuvre muséographique. Les acteurs-témoins y contribuent par leurs souvenirs restitués la plupart du temps en image filmée dans l'exposition. Ils concourent aussi par le prêt de documents et d'objets caractéristiques de leurs modes de vie et de leurs cultures. Ces pièces ne se trouvent généralement pas dans les collections, car elles ne répondent pas au canon habituel de nos musées. Ce sont, à titre d'exemple, des photos de famille ou encore la machine à coudre d'une couturière d'origine italienne. Rien de sensationnel en apparence et pourtant...

\section{Qu'ont en commun ces expositions?}

Au-delà du travail d'histoire et de mémoire, le musée souhaite souligner les apports de ces populations migrantes au territoire sur lequel elles se sont fixées. En effet, le phénomène migratoire est rarement perçu de manière positive par les sociétés. De fait, le propos de ces expositions est également de déconstruire des préjugés souvent négatifs qui apparaissent avec l'évocation du migrant, quelles que soient ses origines. Elles sont aussi là, et ce n'est pas le moindre de leurs objectifs, afin de rappeler le travail qu'il faut accomplir pour mettre à bas la xénophobie et le racisme qui ont accompagné de tout temps la présence de l'étranger. Avec toute l'humilité qu'il convient de garder, on peut espérer que ces expositions contribuent à changer ce genre de perceptions. S'il est complexe, en effet, de mesurer leur impact en termes de fréquentation dans un lieu qui abrite concomitamment plusieurs expositions, l'un des succès de la démarche se manifeste par la présence des populations les plus concernées. Cette présence traduit ainsi une véritable appropriation de la part d'individus et de groupes qui, parfois, ont franchi les portes du musée pour la première fois. Le Musée dauphinois souhaite poursuivre à l'avenir ce cycle d'expositions, car elles participent pleinement au rôle social qu'il s'est assigné. 